\title{
Correction to: Supervised Classification of White Matter Fibers Based on Neighborhood Fiber Orientation Distributions Using an Ensemble of Neural Networks
}

Devran Ugurlu, Zeynep Firat, Ugur Ture and Gozde Unal

\section{Correction to:}

Chapter "Supervised Classification of White Matter Fibers Based on Neighborhood Fiber Orientation Distributions Using an Ensemble of Neural Networks" in: E. Bonet-Carne et al. (eds.), Computational Diffusion MRI, Mathematics and Visualization, https://doi.org/10.1007/978-3-030-05831-9_12

The original version of the chapter was inadvertently published with incorrect affiliation of the corresponding author "Gozde Unal" as "Sabanci University, Istanbul Technical University, Istanbul, Turkey" in Chapter 12. This has now been corrected to "Istanbul Technical University, Istanbul, Turkey". The erratum chapter has been updated with the change. 